Article

\title{
Magnetoelectrochemistry and Asymmetric Electrochemical Reactions
}

\author{
Suryakant Mishra ${ }^{1, *(\mathbb{D}}$, Marzia di Marzio ${ }^{2}$, Roberto Giovanardi ${ }^{2}$ and Francesco Tassinari ${ }^{1}$ \\ 1 Department of Chemical and Biological Physics, Perlman Building, Weizmann Institute of Science, \\ Rehovot 761000, Israel; francesco.tassinari@weizmann.ac.il \\ 2 Department of Engineering “Enzo Ferrari", University of Modena and Reggio Emilia, 41125 Modena, Italy; \\ marzi.jtg@gmail.com (M.d.M.); roberto.giovanardi@unimore.it (R.G.) \\ * Correspondence: suryakant.mishra@weizmann.ac.il
}

Received: 23 August 2019; Accepted: 13 December 2019; Published: 18 December 2019

check for updates

\begin{abstract}
Magnetoelectrochemistry is a branch of electrochemistry where magnetic fields play a vital role in the oxidation and reduction process of the molecules. When it comes to spin-dependent electrochemistry (SDE), becomes a new paradigm. This work presents electrochemical response during the "chiral imprinting" on working electrodes and the effects of potentiostatic and galvanostatic methods. We explore the use of the SDE concept, which is implemented for chiral-ferromagnetic (CFM) hybrid working electrodes, and we compare various electrochemical parameters affecting the quality of deposition. We electrochemically co-deposited nickel ( $\mathrm{Ni}$ ) with a chiral compound (tartaric acid) in its enantiopure forms ( $\mathrm{L}$ and $\mathrm{D}$ ), which allows us to obtain a chiral co-deposited nickel-tartaric acid (Ni-LTA or Ni-DTA) working electrode.
\end{abstract}

Keywords: asymmetric reaction; chiral imprinting; electrodeposition; chiral nickel

\section{Introduction}

The physics behind electron transfer through a helical path is quite fascinating and is currently attracting attention of scientist [1-3]. Electron transfer through the chiral system is spin selective, based on the helicity of chiral system [4-6]. Interplay between charge transmission, chirality, and magnetism is based on the fundamental science which is yet to be exploited. Although there are some theories which explain the mechanism such as the effect of spin orbital coupling (SOC), which is higher when the carbon chain is helical and, due to this, molecules are able to do spin filtering [7-9]. Moreover, besides its intrinsically intriguing aspects, a number of practical applications are here involved, for instance, enantio-recognition is one fundamental aspect of the unfolded problem of homochirality in nature $[10,11]$. Enantio-selectivity in electrochemistry has been seen in a few cases during high-quality electrode preparation. Indeed, chiral surfaces are obtained exploiting a number of different methodologies, such as: adsorption of chiral organic compounds using a self assembly monolayer (SAM), chiral imprinted electrode surfaces by chiral templates or chiral liquid crystals, or chiral metal surfaces by application of a rotating high-intens magnetic field [12]. Breaking chiral symmetry using magnetic fields, provides a framework where chiral molecules are used as a spin filter. Using this effect in electrochemistry, with redox reactions based on spin dependent electron transfer, makes it more efficient [13]. Some applications like water splitting and magnetoresistance are demonstrated in previous reports using such systems [14,15].

Recently, electrodeposition technique has been found to be a useful tool to grow organic and inorganics materials due to its ease of modifying morphology by controlling the current and voltage [16,17]. Present work demonstrates important parameters involve to grow chiral electrode of inorganic material in the presence of magnetic field and organic chiral template within the electrolytic solution. 


\section{Experimental Section}

Chemicals: L-tartaric acid (LTA) and D-tartaric acid (DTA) enantiomers were purchased from Sigma Aldrich and used without further purifications. The nickel electrodeposition was carried out using electrolytic solution of $150 \mathrm{~g} / \mathrm{L}$ of nickel sulphate, $60 \mathrm{~g} / \mathrm{L}$ nickel chloride, and $37 \mathrm{~g} / \mathrm{L}$ boric acid, $\mathrm{pH}=5$. A chiral imprinted ferromagnetic electrode (CFM) is obtained by addition of $0.1 \mathrm{M}$ LTA (or DTA) with reagent solution, giving the relevant Ni-LTA or Ni-DTA electrodes.

Electrochemical set-up: Chronoamperometry was used for electrodeposition under a potentiostatic regime at $-1.4 \mathrm{~V}$ for $900 \mathrm{~s}$ total electrodeposition time. Electrochemical measurements were performed using both Autolab PGSTAT 128N and CHI660A potentiostats, employing a typical three-electrode electrochemical cell. In a special kind of arrangement where $\mathrm{Ni}$ is electrodeposited in the presence of magnetic field: a Teflon cell, featuring a hole $(0.8 \mathrm{~cm}$ diameter $)$ in the bottom, was used in a vertical configuration where the Ni-on-the-magnet working electrode was tightened from below, a Teflon ring was used to avoid solution leakage from the cell. Thus, the Ni surface on the magnet was used as working electrodes, while platinum wire and $\mathrm{Ag} / \mathrm{AgCl}$ sat, were used as counter (CE) and reference electrodes (RE). Control experiments, to check the quality of the nickel surface obtained via electrodeposition on the magnet, were checked using scanning electron microscopy given in supporting information. A $0.1 \mathrm{M} \mathrm{KOH}$ aqueous solution was used as the base electrolyte in all electrochemical measurements. Neodymium permanent magnets of 0.05 Tesla were used for magnetic field.

\section{Result Discussion}

Figure 1a represents the chrono-amperometric response of chiral imprinting of $\mathrm{Ni}$ in the presence of $\mathrm{D}$ and $\mathrm{L}$ tartaric acid. The current is shown as a function of time, namely chronoamperometric measurement, while Figure $1 \mathrm{~b}$ shows the same measurement made under the galvanostatic regime. Notably, pure nickel plating is characterized by rather flat curves under every deposition mode, without any important or sudden change of the electrochemical conditions. Indeed, whereas the curve showed irregular progressing or spikes the resulting coating manifested quite evident defects. No particular deviations from the conventional nickel deposition were observed under potentiostatic settings, save the presence of some ripple in the current vs. time pattern when depositing from the chiral bath. A possible explanation relies on the possibility that the organic compound acts as a sort of complexing agent introducing further steps in the $\mathrm{Ni}^{2+}$ reduction mechanism thus modifying the overall charge transfer process. More remarkable distinctions arise under current control electrodeposition. We compare the relevant results in Figure 2.

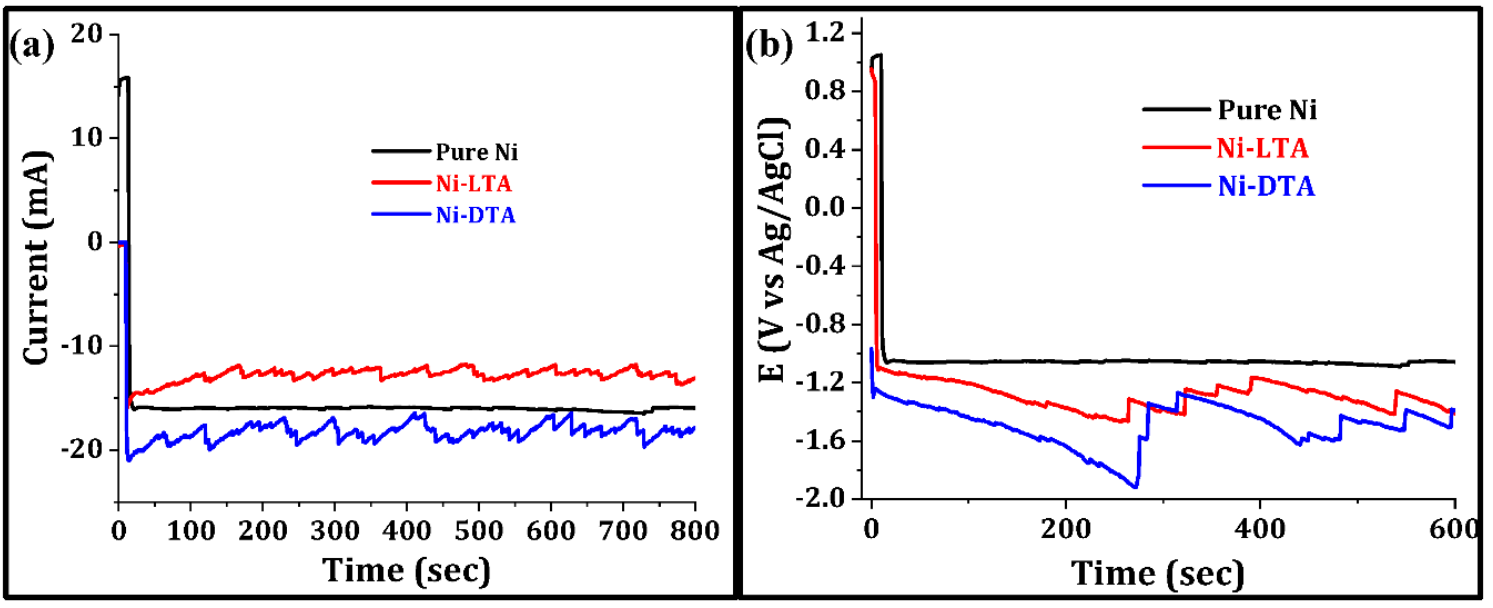

Figure 1. (a) Potentiostat (b) galvanostatic deposition chrono-amperometric response of Ni deposition on evaporated Au surface of pure Ni, Ni-L-tartaric acid (LTA) and Ni-D-tartaric acid (DTA). 


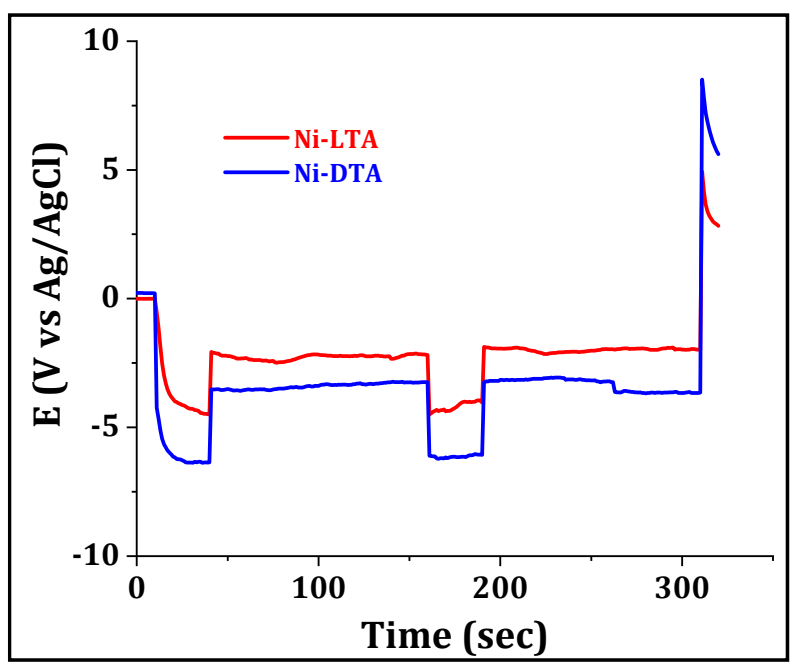

Figure 2. Comparison of low pulsed current deposition by potential steps of Ni-LTA (red) and Ni-DTA (blue) on glassy carbon electrode.

Under galvanostatic technique deposition, the potential of the electrochemical system is variable. In growing the film from baths, where two different enantiomers of the same compound were added, we observed that the potential adjusts itself at values about $2 \mathrm{~V}$ apart (Figure 2); the same outcome was found when the electroplating is carried on in pulse mode on glassy carbon. We also observed a difference of $200 \mathrm{mV}$ in depositing the same type of chiral imprinted film on the two sides of a permanent magnet (Figure 3).

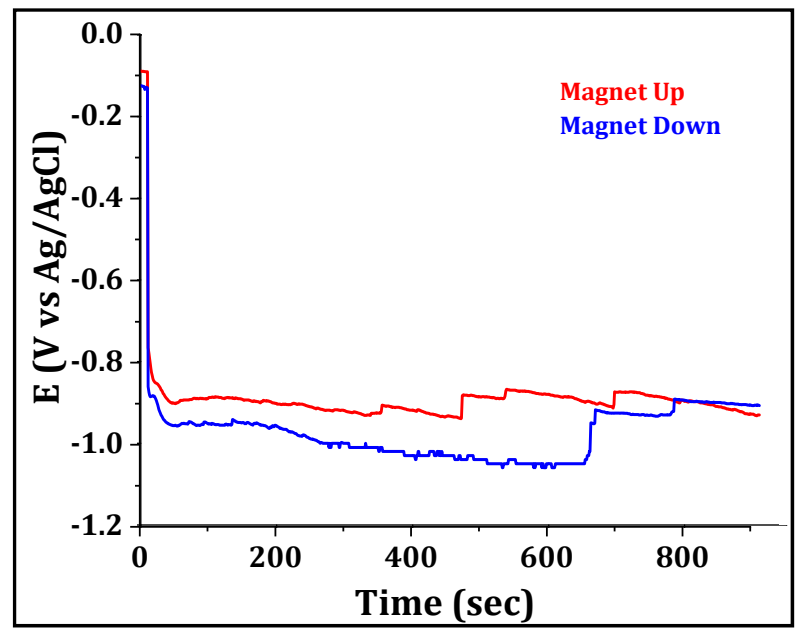

Figure 3. Ni-DTA low current galvanostatic deposition on the up (red) side of the magnet and on the down (blue) side.

More negative potential values are registered when depositing the D-enantiomer imprinted coating with respect to the L-imprinted one; analogously, lower potentials are found when depositing on the "down" side of the magnet (south pole of the magnet toward the surface) and more positive values correspond to the deposition on the "up" side of the magnet (north pole of the magnet toward the surface). The latter situation is likely a consequence of the fact that for ferromagnetic metals under the influence of a magnetic field, due to the modification in the density of states (DoS), there is a shift in the chemical potential that manifests as a shift in electrode potential. 


\section{Morphological Analysis}

We used scanning electron microscopy (SEM) and energy dispersive x-ray spectroscopy (EDS) to estimate the thickness of the film raised under the different electrodeposition modes, and optical microscopy images were taken (given in supporting information). The results are summarized in Table 1:

Table 1. Electrodeposited film thickness estimation from optical microscope imaging.

\begin{tabular}{ccccc}
\hline S. No. & $\begin{array}{c}\text { Deposition Time } \\
\text { (seconds): Pure Ni }\end{array}$ & $\begin{array}{c}\text { Thickness } \\
(\mu \mathrm{m})\end{array}$ & $\begin{array}{c}\text { Deposition Time } \\
\text { (seconds): Ni-LTA }\end{array}$ & Thickness $(\boldsymbol{\mu m})$ \\
\hline 1. & 600 & 9 & 600 & 7.5 \\
2. & 900 & 13.5 & 900 & 8 \\
3. & 1200 & 15 & 1200 & 6.4 \\
\hline
\end{tabular}

Thickness, in the case of conventional nickel electrodeposition, is linearly dependent on deposition time, as expected. Though, the chiral imprinting seems to have a role in limiting the metal reduction on the electrode with film thickness stable around close values even showing a decrease despite the longer experimental time. In sample preparation electrodeposition time of $600 \mathrm{~s}$ and $900 \mathrm{~s}$ were selected as a compromise between deposition time length and a suitable layer growth. Most importantly, the longer the current path (i.e., the distance "travelled" by an electron) through the material the higher will be the probability to lose spin coherence and so the possibility to observe the spin filtering effect.

Nickel potentiostatic deposition from the classical Watts bath leads to a rather fine structure composed of even distributed metallic spheres of about $1 \mu \mathrm{m}$ diameter (Figure S3 in SI). The Ni layer grown in low current galvanostatic conditions has even a finer structure (Figure S4 in SI), while a conventional galvanostatic deposition leads to grains of different morphology, in the range of 1-2 $\mu \mathrm{m}$.

Minor impurities that are detected and visible as point defects in the SEM images are due to the electrodeposition bath drying on the surface. Indeed, microanalysis reveals small amounts of chlorine and sulphur, which constitute the nickel salts from which the metal is reduced. A further chemical analysis collected in correspondence of a visible impurity on the metallic structure (not shown here) has confirmed that. Table 2 summarize the impurity in case of pure $\mathrm{Ni}$ and it appears that the galvanostatic technique produces more metallic films, as suggested from the very low percentages of detected oxygen. On the contrary a higher amount of carbon is found. The pure nickel depositions have been performed from the chiral imprinted bath under the same settings. The microstructure is characterized and presented in the following.

Table 2. Energy dispersive x-ray spectroscopy (EDS) microanalysis result of pure Ni depositions.

\begin{tabular}{|c|c|c|c|c|c|c|}
\hline S. No. & Technique & $\mathrm{Ni} \%$ & $\mathrm{C} \%$ & $\mathrm{O} \%$ & $\mathrm{Cl} \%$ & $\mathrm{~S} \%$ \\
\hline 1. & Ni potentiostatic & 75 & 10.35 & 13.78 & 0.62 & 0.26 \\
\hline 2. & $\begin{array}{l}\text { Ni low current } \\
\text { galvanostatic }\end{array}$ & 80.08 & 17.26 & 2.65 & - & - \\
\hline 3. & Ni galvanostatic & 83.28 & 16.72 & - & - & - \\
\hline
\end{tabular}

Potentiostatic Ni-LTA deposit exhibits larger grains with respect to its equivalent basic nickel and the spherical form has partially been substituted by edges (Figure S5). Low current depositions give rise to particles in the range of $0.5-1 \mu \mathrm{m}$ (Figure S6) analogously to what was observed in the case of the absence of organic compounds, this is consistent with the fact that the low current setting does not allow for an effective chiral imprinting. The microstructure grown under galvanostatic mode corresponds to the one found for the pure nickel, apart for a more spherical morphology of the grains (Figure S3).

EDS chemical investigation, visible in Table 3, confirms an enhanced presence of C, especially in the case of the potentiostatic deposition where the carbon percentage is found to be twice with 
respect to the achiral (no organic compound added) electrode, demonstrating that chiral imprinting has been successfully achieved. Small percentages of Au are reported by the microanalysis of the low current electrodeposited Ni-LTA film, clearly coming from the substrate, suggesting that the layer thickness could be so small that signal from the evaporated Au plating could be detected too. The lower current intensities used for galvanostatic plating are probably not sufficient to grow a deposit thick enough. Furthermore, the low percentage of carbon together with the absence of oxygen suggest that enantiomer molecules were not incorporated in the film in this case. Such deposition is thus found useful to obtain ultrafine structured layers, where the mirror-like appearance is probably due to very low thickness and perfect adherence to the substrate, but failing to co-deposit a significant amount of organic chiral compound. This low current mode can be assimilated to a sort of an underpotential deposition, in accordance with the fact that, throughout the plating, the potential adjusts itself at values much more positive than $-1.4 \mathrm{~V}$ set in potentiostatic mode, as can be seen in the electrodeposition plots reported in Figure 3. Very small impurities are present in the galvanostatic sample, again coming from the electrodeposition solution; in this case, crusts of dried drops are clearly visible in the relative SEM image taken at 5000× (Figure S2a of SI). Finally, the electroplated films prepared under the magnetic field, to ensure a significant impact of the electrodeposition potential on both microstructure and chemical composition of the nickel deposition and it summarized in Table 4.

Table 3. EDS microanalysis result of Ni-LTA depositions.

\begin{tabular}{|c|c|c|c|c|c|c|c|}
\hline S. No. & Technique & $\mathrm{Ni} \%$ & $\mathrm{C} \%$ & $\mathrm{O} \%$ & $\mathrm{Au} \%$ & $S$ & $\mathrm{Cl}$ \\
\hline 1. & Ni-LTA potentiostatic & 74.54 & 20.22 & 5.24 & - & - & - \\
\hline 2. & $\begin{array}{l}\text { Ni-LTA low current } \\
\text { galvanostatic }\end{array}$ & 82.90 & 16.87 & - & 0.23 & - & - \\
\hline 3. & Ni-LTA galvanostatic & 65.42 & 15.45 & 17.74 & - & 0.61 & 0.78 \\
\hline
\end{tabular}

Table 4. EDS microanalysis result of Ni-LTA depositions in presence of external magnetic field.

\begin{tabular}{ccccc}
\hline S. No. & Technique & $\mathbf{N i} \%$ & $\mathbf{C} \%$ & $\mathbf{O} \%$ \\
\hline 1. & Ni-LTA mag UP & 69.85 & 26.29 & 3.86 \\
2. & Ni-LTA mag & 71.05 & 21.05 & 7.90 \\
\hline
\end{tabular}

The deposits grown in the presence of the external magnetic field essentially coincide with the ones that are raised under potentiostatic conditions, except for a slight increase of the grains dimension (Figures S7 and S8 of SI). The presence of a permanent magnet affects the deposition in view of the Lorentz force exerted on metal ions, which results in a slight improvement of the reactants diffusion and in turn in a decrease of the diffusion layer distance. The deposition has been carried positioning the magnet polarized surface just under the cell, that is, with the field lines parallel to the current flow, being the working electrode (WE) at the bottom of the cell and the counter electrode (CE) immersed vertically in the plating solution; nevertheless, the field lines are not strictly perpendicular to the WE but possess a certain rake angle. As a consequence, embedding of the organic molecules in the film seem to be more effective according to the quantitative analysis. In particular, the carbon percentage is larger with respect to both classical and chiral imprinted depositions, of course this is accompanied with a diminishing nickel fraction.

\section{Conclusions}

Magnetic field dependent electrodeposition of chiral $\mathrm{Ni}$ is one step to proving the importance of magnetoelectrochemistry as a technique that plays a significant role in initiating the asymmetric reaction for the induction of chirality. We can conclude some important insights. Using the pulsed mode of the potentiostat and galvanostat techniques gives an interesting chiral system with uniform 
surface morphology. A prepared Ni surface can be further functionalized for spin dependent electrochemistry [18].

Supplementary Materials: The following are available online at http://www.mdpi.com/2312-7481/6/1/1/s1.

Author Contributions: S.M. and F.T. proposed idea, R.G. helped in experimental plan, S.M. and M.d.M. performed all the experiments. S.M. wrote the manuscript. All authors have read and agreed to the published version of the manuscript.

Funding: This research received no external funding.

Acknowledgments: The authors acknowledge research support from the University of Modena and Reggio Emilia (Department of Engineering 'Enzo Ferrari') and the Weizmann Institute of Science (WIS). Ron Naaman (WIS), is gracefully acknowledged for the deep discussion, ideas, and encouragement concerning the application and development of spin-dependent electrochemistry.

Conflicts of Interest: The authors declare no conflict of interest.

\section{References}

1. Ray, K.; Ananthavel, S.P.; Waldeck, D.H.; Naaman, R. Asymmetric Scattering of Polarized Electrons by Organized Organic Films of Chiral Molecules|Science. Available online: https://science.sciencemag.org/ content/283/5403/814 (accessed on 22 August 2019).

2. Kelley, S.O.; Barton, J.K. Electron Transfer Between Bases in Double Helical DNA|Science. Available online: https://science.sciencemag.org/content/283/5400/375 (accessed on 22 August 2019).

3. Göhler, B.; Hamelbeck, V.; Kettner, M.; Markus, T.Z.; Hanne, G.F.; Vager, Z.; Adams, R.; Zacharias, H. Spin Selectivity in Electron Transmission Through Self-Assembled Monolayers of Double-Stranded DNA|Science. Available online: https://science.sciencemag.org/content/331/6019/894 (accessed on 22 August 2019).

4. Kumar, A.; Capua, E.; Fontanesi, C.; Carmieli, R.; Naaman, R. Injection of Spin-Polarized Electrons into a AlGaN/GaN Device from an Electrochemical Cell: Evidence for an Extremely Long Spin Lifetime. ACS Nano 2018, 12, 3892-3897. [CrossRef] [PubMed]

5. Mishra, S.; Poonia, V.S.; Fontanesi, C.; Naaman, R.; Fleming, A.M.; Burrows, C.J. Effect of Oxidative Damage on Charge and Spin Transport in DNA. J. Am. Chem. Soc. 2019, 141, 123-126. [CrossRef] [PubMed]

6. Kumar, A.; Capua, E.; Kesharwani, M.K.; Martin, J.M.L.; Sitbon, E.; Waldeck, D.H.; Naaman, R. Chirality-Induced Spin Polarization Places Symmetry Constraints on Biomolecular Interactions. Proc. Natl. Acad. Sci. USA 2017, 114, 2474-2478. [CrossRef] [PubMed]

7. Guo, A.-M.; Sun, Q. Spin-Selective Transport of Electrons in DNA Double Helix. Phys. Rev. Lett. 2012, 108, 218102. [CrossRef] [PubMed]

8. Guo, A.-M.; Sun, Q.-F. Spin-Dependent Electron Transport in Protein-like Single-Helical Molecules. Proc. Natl. Acad. Sci. USA 2014, 111, 11658-11662. [CrossRef] [PubMed]

9. Michaeli, K.; Kantor-Uriel, N.; Naaman, R.; Waldeck, D.H. The Electron's Spin and Molecular Chirality-How Are They Related and How Do They Affect Life Processes? Chem. Soc. Rev. 2016, 45, 6478-6487. [CrossRef] [PubMed]

10. Banerjee-Ghosh, K.; Dor, O.B.; Tassinari, F.; Capua, E.; Yochelis, S.; Capua, A.; Yang, S.-H.; Parkin, S.S.P.; Sarkar, S.; Kronik, L.; et al. Separation of Enantiomers by Their Enantiospecific Interaction with Achiral Magnetic Substrates. Science 2018, 360, 1331-1334. [CrossRef] [PubMed]

11. Tassinari, F.; Steidel, J.; Paltiel, S.; Fontanesi, C.; Lahav, M.; Paltiel, Y.; Naaman, R. Enantioseparation by Crystallization Using Magnetic Substrates. Chem. Sci. 2019, 10, 5246-5250. [CrossRef] [PubMed]

12. Alvarez, S.; Alemany, P.; Avnir, D. Continuous Chirality Measures in Transition Metal Chemistry. Chem. Soc. Rev. 2005, 34, 313-326. [CrossRef] [PubMed]

13. Gazzotti, M.; Arnaboldi, S.; Grecchi, S.; Giovanardi, R.; Cannio, M.; Pasquali, L.; Giacomino, A.; Abollino, O.; Fontanesi, C. Spin-Dependent Electrochemistry: Enantio-Selectivity Driven by Chiral-Induced Spin Selectivity Effect. Electrochim. Acta 2018, 286, 271-278. [CrossRef]

14. Mtangi, W.; Tassinari, F.; Vankayala, K.; Vargas Jentzsch, A.; Adelizzi, B.; Palmans, A.R.A.; Fontanesi, C.; Meijer, E.W.; Naaman, R. Control of Electrons' Spin Eliminates Hydrogen Peroxide Formation During Water Splitting. J. Am. Chem. Soc. 2017, 139, 2794-2798. [CrossRef] [PubMed] 
15. Mtangi, W.; Kiran, V.; Fontanesi, C.; Naaman, R. Role of the Electron Spin Polarization in Water Splitting. J. Phys. Chem. Lett. 2015, 6, 4916-4922. [CrossRef] [PubMed]

16. Mishra, S.; Yogi, P.; Sagdeo, P.R.; Kumar, R. TiO2-Co3O4 Core-Shell Nanorods: Bifunctional Role in Better Energy Storage and Electrochromism. ACS Appl. Energy Mater. 2018, 1, 790-798. [CrossRef]

17. Mishra, S.; Fontanesi, C. Combined Effect of Organic-Inorganic Heterostructure to Enhance Electrochemical Capacitance. Mater. Chem. Phys. 2019, 238, 121943. [CrossRef]

18. Fontanesi, C.; Tassinari, F.; Parenti, F.; Cohen, H.; Mondal, P.C.; Kiran, V.; Giglia, A.; Pasquali, L.; Naaman, R. New One-Step Thiol Functionalization Procedure for Ni by Self-Assembled Monolayers. Langmuir 2015, 31, 3546-3552. [CrossRef] [PubMed]

(C) 2019 by the authors. Licensee MDPI, Basel, Switzerland. This article is an open access article distributed under the terms and conditions of the Creative Commons Attribution (CC BY) license (http://creativecommons.org/licenses/by/4.0/). 